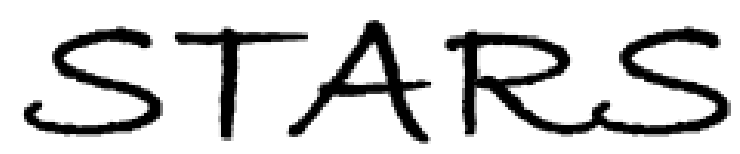

University of Central Florida

STARS

Faculty Bibliography 2000s

Faculty Bibliography

$1-1-2006$

\title{
Method of images in optical discrete systems
}

Konstantinos G. Makris

University of Central Florida

Demetrios N. Christodoulides

University of Central Florida

Find similar works at: https://stars.library.ucf.edu/facultybib2000

University of Central Florida Libraries http://library.ucf.edu

This Article is brought to you for free and open access by the Faculty Bibliography at STARS. It has been accepted for inclusion in Faculty Bibliography 2000 s by an authorized administrator of STARS. For more information, please contactSTARS@ucf.edu.

\section{Recommended Citation}

Makris, Konstantinos G. and Christodoulides, Demetrios N., "Method of images in optical discrete systems" (2006). Faculty Bibliography 2000s. 6395.

https://stars.library.ucf.edu/facultybib2000/6395

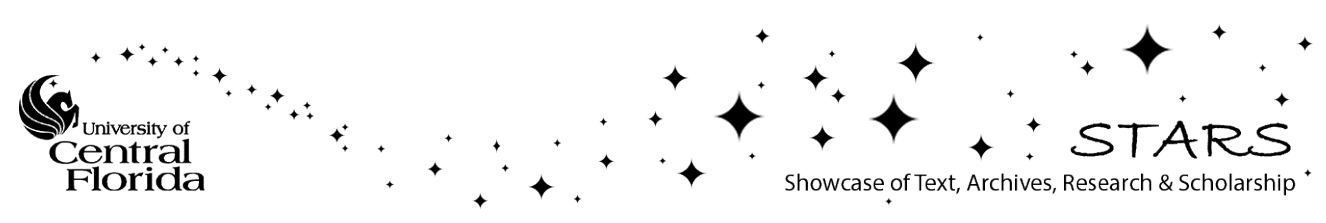




\title{
Method of images in optical discrete systems
}

\author{
Konstantinos G. Makris and Demetrios N. Christodoulides \\ College of Optics/CREOL, University of Central Florida, Orlando, Florida 32816, USA
}

(Received 4 November 2005; published 23 March 2006)

\begin{abstract}
We show that optical wave propagation in discrete boundary geometries can be effectively analyzed using the method of images. Such semi-infinite and finite discrete systems include, for example, waveguide arrays as well as coupled resonator optical waveguides. In the linear domain, the diffraction properties of one- and two-dimensional bounded array structures are considered in detail. The possibility of using the method of images to numerically investigate nonlinear semi-infinite lattices is also discussed.
\end{abstract}

DOI: 10.1103/PhysRevE.73.036616

PACS number(s): 42.65.Tg, 42.65.Sf, 42.82.Et

\section{INTRODUCTION}

The discrete coupling or tunneling process between periodically arranged potential wells is a fundamental topic that has been extensively investigated in many branches of physics. In optics, arrays of weakly coupled waveguides and resonators are prime examples of such systems where the coupling dynamics can be directly observed and investigated $[1,2]$. Periodic array structures are typically comprised from single-mode waveguides that are coupled to each other through the evanescent tails of adjacent guided fields [2]. Likewise, the transport dynamics in periodic chains of microcavities (coupled resonator waveguides or CROWs) follow similar rules [3]. In these configurations, linear mode coupling leads to energy redistribution among the elements of the array, a mechanism better known as discrete diffraction. The problem of discrete diffraction in infinite optical arrays was first analytically solved by Jones in 1965 [1]. This was done by explicitly obtaining the impulse response of the infinite chain in terms of Bessel functions. This behavior was subsequently observed in one-dimensional (1D) AlGaAs waveguide arrays by several experimental groups [4,5]. Lately, diffraction in two-dimensional discrete systems has also been observed in femtosecond laser induced waveguide arrays [6] and in optically induced photorefractive lattices [7].

Under nonlinear conditions (when the material is nonlinear and at high excitation powers), a self-localized nonlinear entity can also be supported by the periodic potential of the array. This nonlinear wave or optical discrete soliton [8] remains invariant during propagation through a balance of diffraction and nonlinearity. Again the discreteness offers a rich spectrum of properties and possibilities that do not exist in the bulk. Modulation instability [9] and discrete/lattice solitons in cubic [5,7-10], photorefractive [11], and quadratic waveguide arrays $[12,13]$ have been examined theoretically, and observed experimentally. In addition, discrete solitons in systems that exhibit nonlocal nonlinearities, such as semiconductor amplifiers and nematic liquid crystals, have been considered $[14,15]$.

Boundaries and surfaces can also introduce new physical features due to the break of the translational symmetry. For example, electromagnetic surface waves are possible along the boundary between two different media (continuous or periodic). Linear and nonlinear surface waves in both bulk and periodic environments have been considered during the past few years in various fields of science [16-22].

Clearly the presence of boundaries can considerably complicate the wave dynamics, even in linear array networks. Unlike infinite discrete systems whose diffraction characteristics have been known for some time $[1,4]$, the corresponding behavior in semi-infinite or finite arrays remains to be explored.

In this paper, we demonstrate that optical wave propagation in discrete boundary geometries can be analyzed using the method of images. This is done by introducing fictitious sources outside the region of interest, in a way similar to the method of images used in other fields like electrostatics [23], mechanics [24], electrodynamics [25], and solid state physics [26]. The proposed method offers several advantages in terms of studying this broad class of problems. More specifically, for certain lattice topologies the use of images leads to closed form solutions, whereas for finite array geometries it greatly simplifies the analysis. In addition, this same technique can be employed to study wave propagation in bounded nonlinear discrete systems, provided that the initial conditions are preserved. This method is elucidated by providing pertinent examples.

\section{SEMI-INFINITE AND FINITE ONE-DIMENSIONAL WAVEGUIDE ARRAYS}

Let us consider a linear semi-infinite array of weakly coupled waveguides, like the one depicted in Fig. 1(a). Within the context of coupled mode theory, wave propaga-

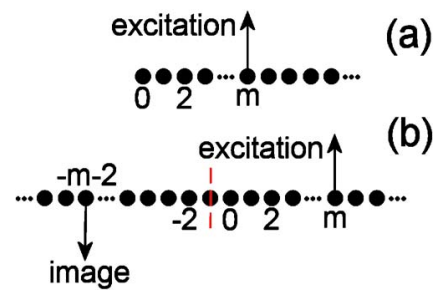

FIG. 1. (Color online) (a) A semi-infinite waveguide array under single site excitation at position $m$, and (b) the equivalent infinite array with the image positioned at the $-(m+2)$ site. The field in the $n=-1$ waveguide is always zero, because of the mirror symmetry. 
tion in such a structure is described by the following equations:

$$
\begin{gathered}
i \frac{d E_{0}}{d z}+\beta E_{0}+c E_{1}=0, \\
i \frac{d E_{n}}{d z}+\beta E_{n}+c\left(E_{n+1}+E_{n-1}\right)=0, \quad \text { for } n \geqslant 1,
\end{gathered}
$$

where $E_{n}$ is the electric modal field in the $n$th waveguide, $\beta$ is the propagation constant, and $c$ is the coupling coefficient. We note that similar equations are applied to describe CROW microcavities in the temporal domain [3]. By utilizing the transformation $E_{n}=a_{n} \exp (i \beta z)$ and by normalizing the propagation distance with respect to the coupling length, i.e., $Z=c z$, Eqs. (1) are rewritten as

$$
\begin{gathered}
i \frac{d a_{0}}{d Z}+a_{1}=0, \\
i \frac{d a_{n}}{d Z}+a_{n+1}+a_{n-1}=0, \quad \text { for } n \geqslant 1,
\end{gathered}
$$

where $a_{n}$ represents the normalized modal amplitude in the $n$th waveguide. In order to study the diffraction properties of this semi-infinite array it is first important to derive its impulse response. The impulse response of this structure is, in fact, the solution of Eqs. (2) under single site excitation, i.e., $a_{n}=A_{0} \delta_{n m}$ (if, for example, site $m$ is initially excited). Following the argument presented later, the diffraction problems in infinite and semi-infinite arrays are related to each other through the method of images. The goal here is to identify an equivalent infinite system in which the half region is governed by Eqs. (2). This can be done by demanding that the field at the site $a_{-1}$ is always zero during propagation $\left[a_{-1}(Z)=0\right]$. This last requirement can only be satisfied if a fictitious source or image with a relative phase difference $\pi$ (with respect to the actual source) is positioned symmetrically around the $n=-1$ site. This is because the antisymmetric conditions used at the input guarantee that $a_{-1}(Z)=0$ for all values of propagation distance $Z$. As a result, the two semi-infinite sections of the equivalent infinite array are decoupled and thus Eqs. (2) hold true in the region of interest $(n \geqslant 0)$. Therefore the study of the semi-infinite array can be carried out by considering the diffraction dynamics in an infinite lattice under appropriate initial conditions. In this case the superposition of the fields emanating from the actual source and the image provide the impulse response of the semi-infinite array. By using the already known impulse response of an infinite array, that is, $a_{n}(Z)=A_{0} i^{n-m} J_{n-m}(2 Z)$ when site $m$ is excited [1], the diffraction problem can then be directly solved. Given that the excitation site is at $\mathrm{m}$, then its image (with respect to the $n=-1$ waveguide) is positioned at the $-(m+2)$ channel, (see Fig. 1(b)). Thus, the diffracted field resulting from the actual excitation site is given by $i^{n-m} J_{n-m}(2 Z)$, whereas that originating from its image is described by $(-1) i^{n+m+2} J_{n+m+2}(2 Z)$. Hence the corresponding impulse response of the semi-infinite array [analytical solution to Eq. (2)] is given in closed form by

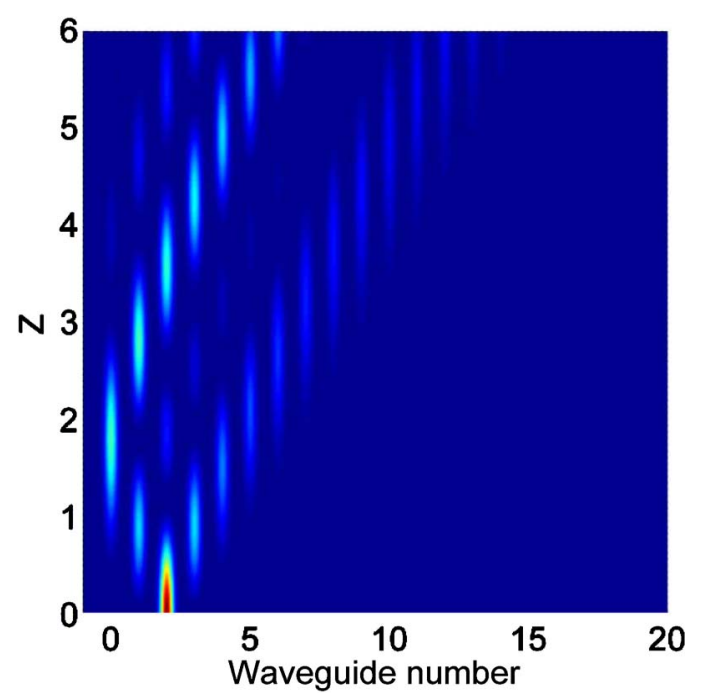

FIG. 2. (Color online) Intensity distribution in a semi-infinite array, when only the channel $n=2$ is excited.

$$
a_{n}(Z)=A_{0}\left[i^{n-m} J_{n-m}(2 Z)+i^{n+m} J_{n+m+2}(2 Z)\right] .
$$

The validity of the method of images in dealing with optical discrete systems can also be formally justified. In particular, by applying $\mathbf{Z}$-transform techniques [10,27,28], one can analytically show (see the Appendix), that an antisymmetric initial condition at $Z=0, a_{-1}(0)=0, a_{n}(0)=-a_{-n-2}(0)$, remains antisymmetric during propagation. This implies that $a_{-1}(Z)=0$, and $a_{n}(Z)=-a_{-n-2}(Z)$ for every value of $Z$. Under such initial conditions, the wave propagation in an infinite waveguide array in the region of interest $n \geqslant 0$, is governed by Eqs. (2) of the semi-infinite array. Since the two structures are described by the same equations and since the solution of the system of ordinary differential equations happens to be unique, we then conclude that the two array systems are mathematically equivalent for $n \geqslant 0$. The discrete diffraction pattern of a semi-infinite array when the channel $n=2$ is initially excited is depicted in Fig. 2.

The method of images can also be employed to study the diffraction in a finite array of $N$ waveguides. Obtaining the impulse response of a finite lattice is more complicated, since this type of structure involves two boundaries. In this case, the problem can be mapped to that of an infinite waveguide array, where the field in the two channels (denoted L and R) located (left and right) at the fictitious edges of the system $(N+1$ sites away from each other) remains always zero. Between these two virtual boundaries, the infinite and the finite array exhibit the same behavior. To find the impulse response of this structure, let us consider the case where a single waveguide site is excited between the $\mathrm{L}$ and $\mathrm{R}$ channels. In order for the field in these waveguides to be always zero, the corresponding images must be appropriately situated in the equivalent infinite array. In particular, a negative image is positioned symmetrically with respect to the virtual site L (see Fig. 3) and another one (negative) with respect to $\mathrm{R}$. These images act as secondary excitation sites and in turn lead to two new positive images. One of these two new images results from the reflection of the secondary image located at the left of $\mathrm{L}$ with respect to the $\mathrm{R}$ site and similarly 


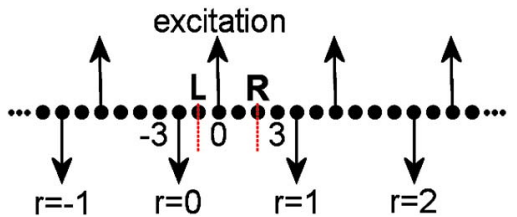

FIG. 3. (Color online) Equivalent infinite array configuration for the case of a finite array of $\mathrm{N}$ elements. For simplicity here, $N=2$. The region of interest lies between the $\mathrm{L}$ and $\mathrm{R}$ waveguide sites. The index $r$ denotes the image pair.

the other from a reflection at $\mathrm{L}$ (see Fig. 3). This process continues indefinitely and the result is an infinite number of positive and negative pairs of images. For illustration purposes, Fig. 3 shows the positions of these pairs of images when $N=2$. Once more, by applying the superposition principle, a closed form expression for the impulse response of an array of $N$ waveguides, can be found. When the $m$ th site of the finite array is excited, the field at the $n$th site is given by the expression

$$
\begin{aligned}
a_{n}(Z)= & A_{0} \sum_{r=-\infty}^{+\infty}\left\{i ^ { - ( 2 N + 2 ) r } \left[i^{n-m} J_{n-m-(2 N+2) r}(2 Z)\right.\right. \\
& \left.\left.-i^{n+m+2} J_{n+m+2-(2 N+2) r}(2 Z)\right]\right\},
\end{aligned}
$$

where $r$ is the image index pair, and $2 N+2$ is the period between the positive or negative images.

The use of the method of images in analyzing finite arrays offers several advantages over other schemes especially when the number of elements $N$ is relatively large. In principle, the impulse response of a finite array can be obtained by considering the projection of the input vector over the supermode eigenvectors of the array [29]. Yet, this latter approach requires summing up $N$ contributions, something that is impractical when $N$ is large. On the other hand, for finite distances, the method of images can provide a solution to this problem, by only keeping a finite number of terms in the Bessel function expansion of Eq. (4). This approximate description will accurately follow the wave dynamics in a finite array as long as the consecutive reflections from the boundary walls correspond the image pair of images (accounted in the truncated expression). Figure 4 shows the discrete diffraction in an $N=5$ array, when the $n=1$ site is excited up to a normalized distance of $Z=5$. These results were obtained using only four pairs of images and are in excellent agreement with the actual response of the system.

\section{LINEAR 2D SEMI-INFINITE WAVEGUIDE ARRAYS}

The method of images can also be extended to analyze semi-infinite two-dimensional periodic structures. In general, wave propagation in an infinite two-dimensional waveguide array is governed by the normalized coupled mode equation,

$$
i \frac{d a_{n, m}}{d Z}+a_{n, m+1}+a_{n, m-1}+a_{n+1, m}+a_{n-1, m}=0
$$

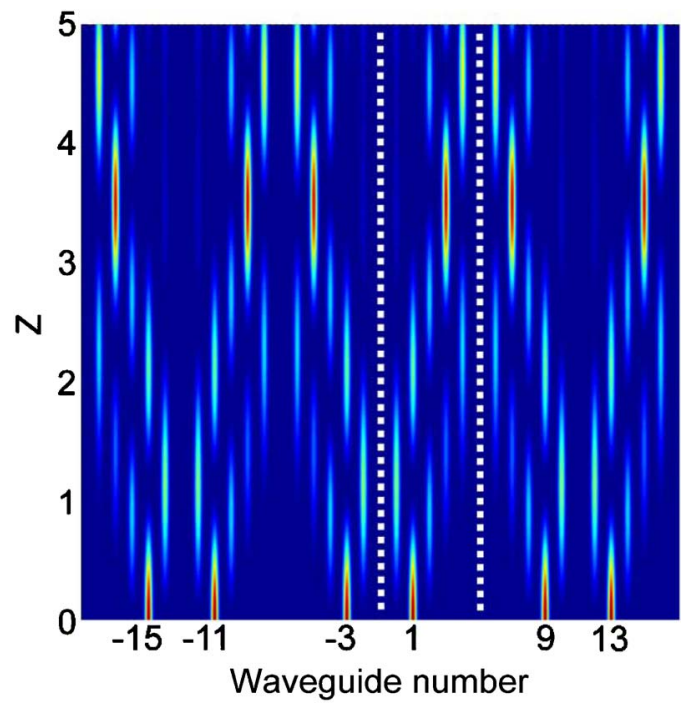

FIG. 4. (Color online) Diffraction pattern in an array of five elements, by using the equivalent infinite array with nine images. The region that corresponds to the finite array is located between the two white dotted lines. The site $n=1$ was initially excited. Here only five images are shown.

where $a_{n, m}$ is the modal amplitude at the $(n, m)$ site, and $Z$ the normalized propagation distance. In deriving Eq. (5), we have assumed negligible diagonal coupling effects, and we have considered only nearest-neighbor interactions. If only one channel is excited at the $(p, q)$ site, i.e., $a_{n, m}(0)$ $=A_{0} \delta_{n p} \delta_{m q}$, the discrete diffraction is described by $a_{n, m}(Z)$ $=A_{0} i^{(n-p)} i^{(m-q)} J_{n-p}(2 Z) J_{m-q}(2 Z)$. Since the impulse response of an infinite 2D lattice is known, closed form solutions can be obtained by applying the method of images in the case of diffraction problems involving boundaries.

A discrete arrangement in a semi-infinite plane is shown in Fig. 5(a). The source in this lattice is positioned at the $(p, q)$ site. Following the rationale of the previous section, the image (with a $\pi$ phase shift) is located at $(-p-2, q)$, and the virtual zero line is at $p=-1$ (see Fig. 5(b)). In this case, the field distribution at the $(n, m)$ site when initially $a_{n, m}(0)=A_{0} \delta_{n p} \delta_{m q}$ is given by

$$
\begin{aligned}
a_{n, m}(Z)= & A_{0}\left\{i^{(n-p)} i^{(m-q)} J_{n-p}(2 Z) J_{m-q}(2 Z)\right. \\
& \left.+i^{(n+p)} i^{(m-q)} J_{n+p+2}(2 Z) J_{m-q}(2 Z)\right\} .
\end{aligned}
$$

Figure 6 demonstrates the intensity pattern in such a semi-infinite lattice topology when the site $(3,0)$ is initially excited.

\section{LINEAR 2D WAVEGUIDE ARRAY ANGULAR SECTORS}

In this section we will use the image method to study $2 \mathrm{D}$ array angular sectors. A $90^{\circ}$ corner is shown in Fig. 7(a). The discrete diffraction resulting from a single excited site can be obtained by considering the equivalent $2 \mathrm{D}$ infinite array under the appropriate initial conditions. In a way similar to that used in electrostatics, in order for the method of images to 

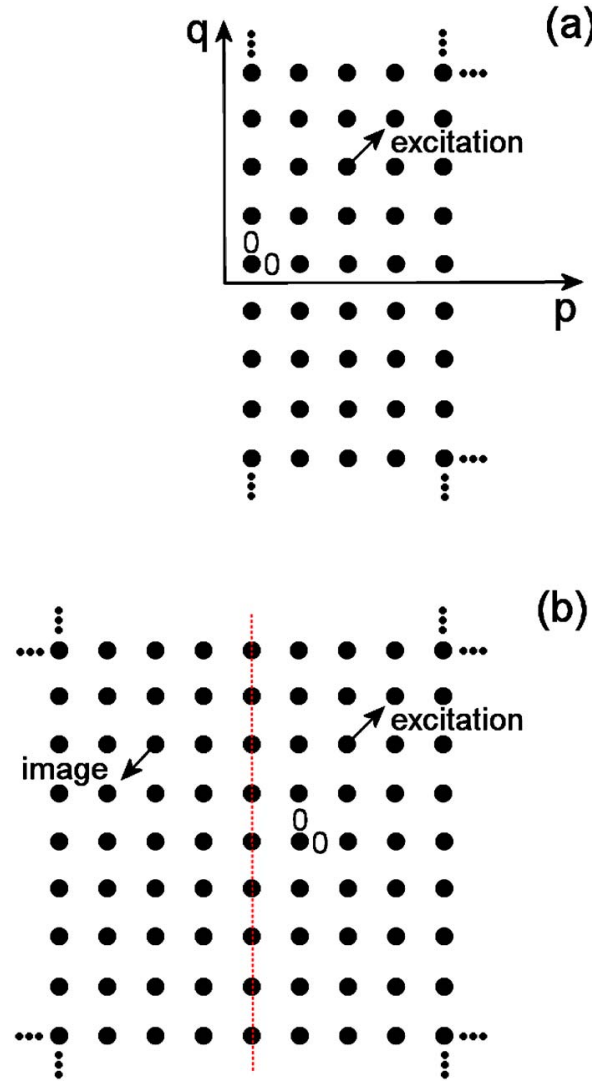

(b)

FIG. 5. (Color online) (a) A semi-infinite two-dimensional lattice where the source is at the $(p, q)$ site, and (b) the equivalent infinite lattice with the source and the corresponding anti-phase image located at $(-p-2, q)$ waveguide channel. The axis of symmetry is illustrated as a dotted line.

work, the field along the two axes of symmetry $p=-1$, and $q=-1$ must be always zero. More specifically, if the excitation occurs at the $(p, q)$ channel in the positive quadrant, the related images are located at the three symmetric positions with respect to the center of the lattice $(-1,-1)$. As depicted in Fig. 7(b), the two negative images $\mathrm{A}$ and $\mathrm{C}$ are situated at $(-p-2, q),(p,-q-2)$, respectively, and the positive image B at $(-p-2,-q-2)$. The two pairs (actual source, image A) and images $(\mathrm{B}, \mathrm{C})$ keep the $p=-1$ axis at zero, while the axis $q=-1$ is at zero because of the other two pairs (A, B) and (excitation, C). By superposing the fields from the actual source and the three images, we obtain the analytical solution for the diffracted field at the $(n, m)$ site (in the positive quadrant) when $a_{n, m}(0)=A_{0} \delta_{n p} \delta_{m q}$, that is,

$$
\begin{aligned}
a_{n, m}(Z)= & A_{0}\left[i^{(n-p)+(m-q)} J_{n-p}(2 Z) J_{m-q}(2 Z)\right. \\
& +i^{(n-p)+(m+q)} J_{n-p}(2 Z) J_{m+q+2}(2 Z) \\
& +i^{(n+p)+(m-q)} J_{n+p+2}(2 Z) J_{m-q}(2 Z) \\
& \left.+i^{(n+p)+(m+q)} J_{n+p+2}(2 Z) J_{m+q+2}(2 Z)\right] .
\end{aligned}
$$

The intensity distribution after a normalized distance of $Z=4$, where only the $(0,1)$ site is initially excited is depicted in Fig. 8.

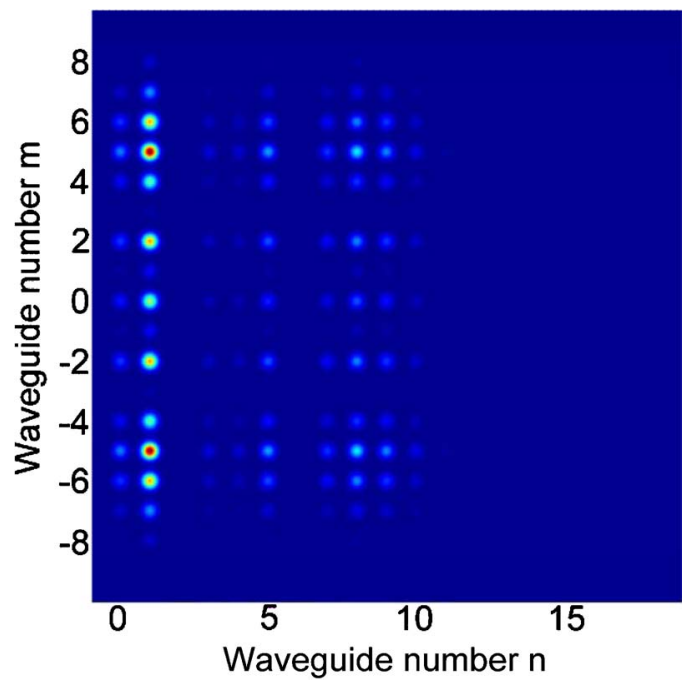

FIG. 6. (Color online) Discrete diffraction in a semi-infinite two dimensional waveguide array for a normalized distance of $Z=3.3$, when only the $(3,0)$ site was initially excited.

Another interesting lattice configuration that can be analytically treated using this technique, is that of a $2 \mathrm{D} 45^{\circ}$ array corner. This lattice sector is contained between the $+45^{\circ}$ axis and the $0^{\circ}$ axis of symmetry (see Fig. 9(a)). In this case, seven images are required in order to keep the four axes $p=-2, q=-1,+45^{\circ}$, and $-45^{\circ}$ degree always at zero (see Fig. 9(b)). When the excited site is located at $(p, q)$ (with $q \leqslant p$ ), then the field at the $(n, m)$ channel is given by the following relation:

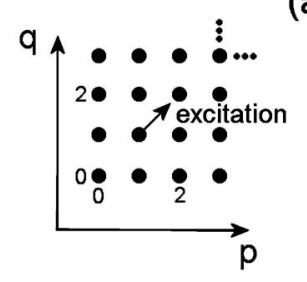

(a)

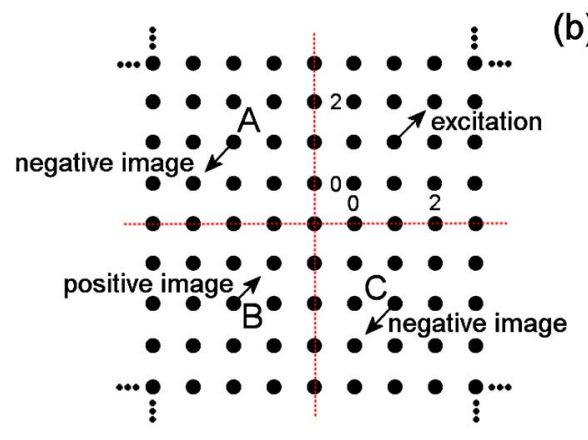

(b)

FIG. 7. (Color online) (a) A two-dimensional lattice angular sector of $90^{\circ}$, where the source is at the $(p, q)$ site and (b) the equivalent infinite two-dimensional lattice with the source and the corresponding three images A, B, C appropriately positioned. The axes of symmetry are shown with dotted lines. 


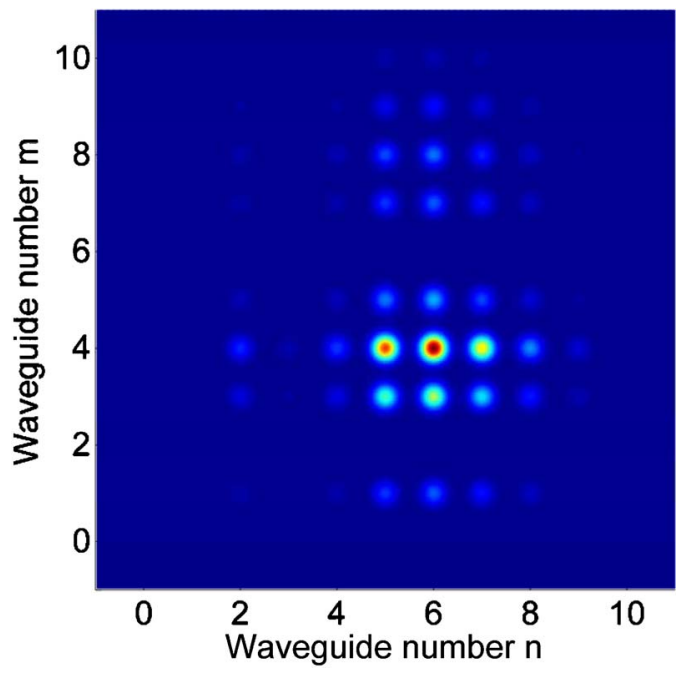

FIG. 8. (Color online) Intensity pattern in two-dimensional $90^{\circ}$ waveguide array corner for a normalized distance of $Z=4$, under a $(0,1)$ waveguide site excitation.

$$
\begin{aligned}
a_{n, m}(Z)= & A_{0}\left\{i ^ { ( n - p ) + ( m - q ) } \left[J_{n-p}(2 Z) J_{m-q}(2 Z)\right.\right. \\
& \left.-J_{n-q+1}(2 Z) J_{m-p-1}(2 Z)\right]+i^{(n+p)+(m-q)} \\
& \times\left[J_{n-q+1}(2 Z) J_{m+p+3}(2 Z)-J_{n+p+4}(2 Z) J_{m-q}(2 Z)\right] \\
& +i^{(n+p)+(m+q)}\left[J_{n+q+3}(2 Z) J_{m+p+3}(2 Z)\right. \\
& \left.-J_{n+p+4}(2 Z) J_{m+q+2}(2 Z)\right]+i^{(n-p)+(m+q)} \\
& \left.\times\left[J_{n-p}(2 Z) J_{m+q+2}(2 Z)-J_{n+q+3}(2 Z) J_{m-p-1}(2 Z)\right]\right\}
\end{aligned}
$$

Figure 10 shows the intensity pattern resulting from the discrete diffraction at $Z=3$, when the site $(0,0)$ has been initially excited.

\section{NONLINEAR WAVEGUIDE ARRAYS}

Thus far, we have only considered linear optical lattices where the superposition principle can be applied in conjunction with the method of images to study their diffraction characteristics. Clearly, if the array is nonlinear such superposition is not allowed. In this case the underlying evolution dynamics are governed by a discrete nonlinear Schrödinger equation [8]. If the nonlinearity is of the Kerr type, then a 1D semi-infinite waveguide array is described by the following equations:

$$
\begin{gathered}
i \frac{d a_{0}}{d Z}+a_{1}+\left|a_{0}\right|^{2} a_{0}=0, \\
i \frac{d a_{n}}{d Z}+a_{n+1}+a_{n-1}+\left|a_{n}\right|^{2} a_{n}=0, \quad \text { for } n \geqslant 1 .
\end{gathered}
$$

Yet, even though the superposition principle is no longer valid (because of nonlinearity) the method of images can still be useful in analyzing nonlinear bounded discrete geometries. For example, discrete surface soliton solutions can be numerically obtained from the equations of an infinite lattice by assuming an antisymmetric or twisted $\left(a_{n}=-a_{-n-2}\right)$ field
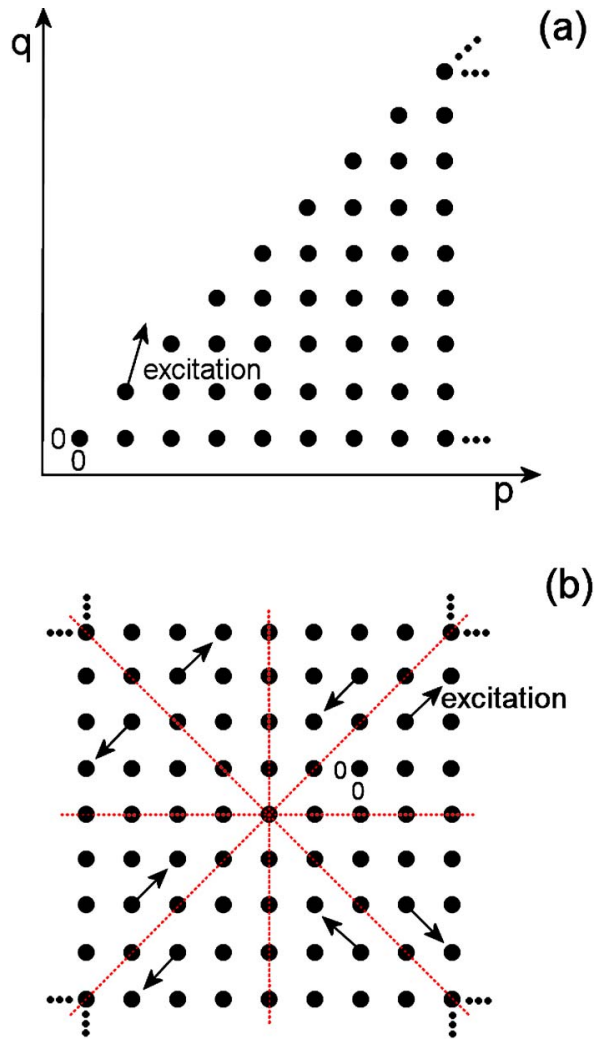

(b)

FIG. 9. (Color online) (a) A two-dimensional lattice angular sector of $45^{\circ}$, where the source is at the $(p, q)$ site, and (b) the equivalent infinite two-dimensional lattice under the excitation of the source and the corresponding seven images. The axes of symmetry are depicted with dotted lines.

profile. Figure 11(a), shows the intensity profile of such a solution and the corresponding power-eigenvalue diagram [22]. In agreement with the Vakhitov-Kolokolov criterion these solutions are only stable for $\mu>2.99$, where $d P / d \mu$ $>0$. On the other hand Fig. 11(b) depicts a twisted soliton solution [30,31], as obtained in an infinite array. Note that

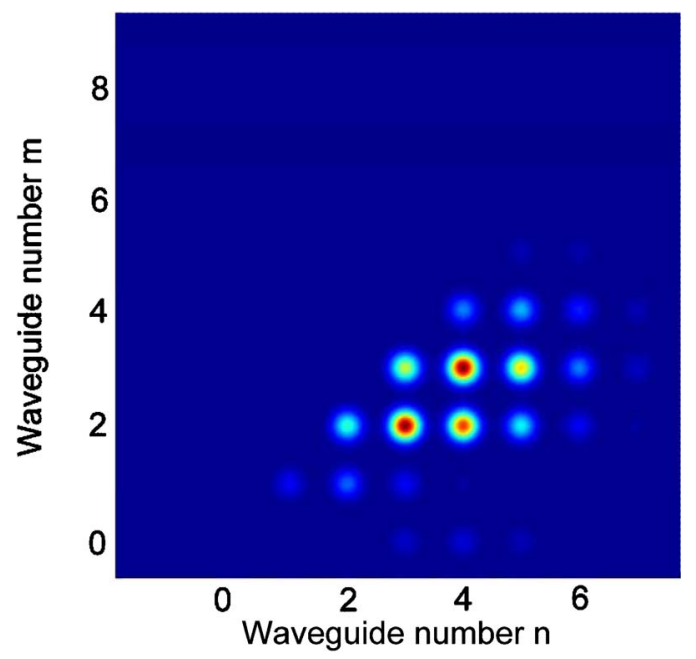

FIG. 10. (Color online) Diffraction evolution in twodimensional $45^{\circ}$ waveguide array angular sector for a normalized distance of $Z=3$. Channel $(0,0)$ has been excited. 

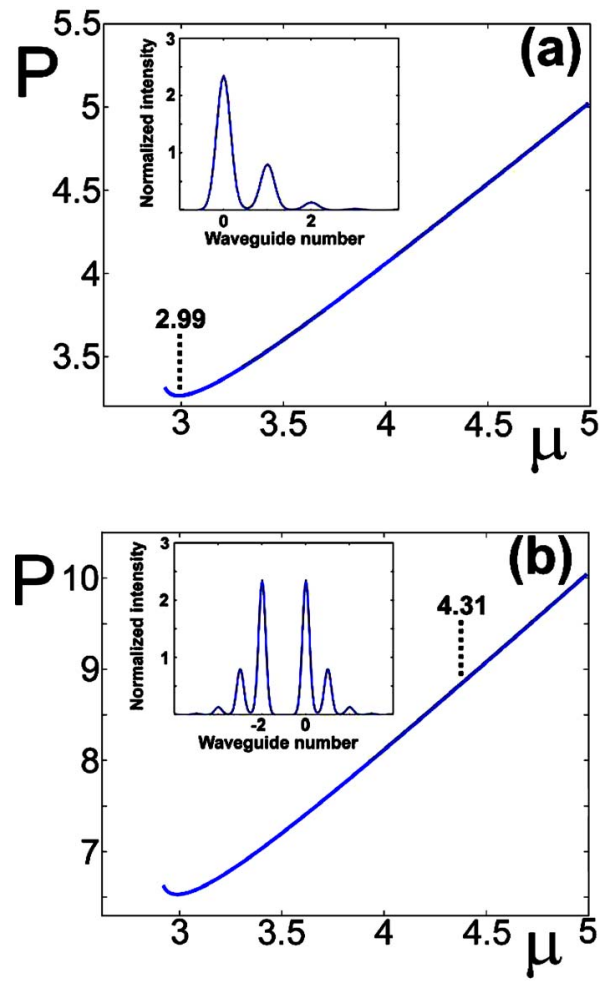

FIG. 11. (Color online) Normalized discrete soliton power $P$ versus the normalized eigenvalue $\mu$, for the cases of (a) a surface soliton in a semi-infinite array, and (b) a twisted soliton in an infinite array. The intensity distribution associated with $\mu=2.93$ surface soliton and twisted soliton are shown in the insets of (a) and (b), respectively.

the assumed antisymmetry makes this problem directly relevant to the semi-infinite case. The solution of Fig. 11(b) exhibits a $P-\mu$ diagram that is in fact a scaled version (by a factor of 2 in terms of power) of that in Fig. 11(a). This should have been expected since the surface soliton solution of the semi-infinite array (inset of Fig. 11(a)) is just the right-hand part of that of the infinite system (inset of Fig. 11(b)). Yet, the stability properties of these two states are very different. The twisted mode of the infinite array happens to be stable for $\mu>4.31$, as opposed to the surface state, which is stable for $\mu>2.99$. This is because these solutions correspond in reality to altogether different physical problems. The former is a surface state whereas the latter is twisted self-trapped mode. We note that quite recently we successfully used this nonlinear version of the method of images in order to simulate the spatiotemporal dynamics of optical pulses in semi-infinite $\mathrm{AlGaAs}$ and $\mathrm{LiNbO}_{3}$ waveguide arrays [32]. This was done by using an "antisymmetric" excitation in an equivalent infinite system, which conserves the condition $a_{-1}(Z)=0$ necessary for this method to work.

\section{CONCLUSIONS}

In this paper, we have shown that optical wave propagation in discrete boundary geometries can be analyzed using the method of images. This was done by introducing fictitious sources outside the region of interest. Analytical solutions in various $1 \mathrm{D}$ and 2D lattice topologies have been obtained. These include, for example, 1D semi-infinite arrays, finite systems, and 2D angular sectors. The use of the method in nonlinear discrete systems was also discussed.

\section{APPENDIX}

Let us consider the normalized wave propagation equation in a infinite waveguide array,

$$
i \frac{d a_{n}}{d Z}+a_{n+1}+a_{n-1}=0 .
$$

By using the $\mathbf{Z}$ transform, we will show here that the mirror symmetry assumed at the input of the array $(Z=0)$ is preserved during propagation. In other words, if $a_{n}(0)=-a_{-n}(0), a_{0}(0)=0$, then $a_{n}(Z)=-a_{-n}(Z), a_{0}(Z)=0$ for every propagation distance $Z$. For convenience, we assume that the zero symmetry line is at $n=0$.

The $\mathbf{Z}$ transform $A(Z)$ of the sequence $\left\{a_{n}\right\}$ and its corresponding inverse transform $a_{n}(Z)$ are defined in the complex domain as $[10,27]$

$$
\begin{gathered}
A(Z)=\sum_{n=-\infty}^{+\infty} a_{n} x^{n}, \\
a_{n}(Z)=\frac{1}{2 \pi i} \oint_{|x|=1} A(Z) x^{-n-1} d x .
\end{gathered}
$$

The $\mathbf{Z}$ transform of Eq. (A1) is

$$
A(Z, x)=A(0, x) \exp \left[i\left(x+x^{-1}\right) Z\right] .
$$

From (A2) and the imposed initial conditions at $Z=0$, the $A(0, x)$ term can be found. More specifically, we get

$$
A(0, x)=\sum_{n=-\infty}^{+\infty} a_{n}(0) x^{n},
$$

therefore

$$
A(0, x)=\sum_{n=1}^{+\infty} a_{n}(0)\left(x^{n}-x^{-n}\right) .
$$

This provides $A(Z, x)$, which is

$$
A(Z, x)=\sum_{n=1}^{+\infty} a_{n}(0)\left(x^{n}-x^{-n}\right) \exp \left[i\left(x+x^{-1}\right) Z\right] .
$$

Now the inverse $\mathbf{Z}$ transform can be applied to the sequences $\left\{a_{n}\right\}$ and $\left\{a_{-n}\right\}$. The sum of these two inverse transforms is

$$
a_{n}(Z)+a_{-n}(Z)=\sum_{n=1}^{+\infty} a_{n}(0) I_{n},
$$

where the complex integral $I_{n}$ is given by 


$$
I_{n}=\frac{1}{2 \pi i} \oint_{|x|=1}\left(x^{2 n}-x^{-2 n}\right) \exp \left[i\left(x+x^{-1}\right) Z\right] \frac{d x}{x} .
$$

By switching to polar coordinates and by using the integral representation of the Bessel function [33], we obtain

$$
\frac{1}{2 \pi i} \oint_{|x|=1} x^{-n} \exp \left[i\left(x+x^{-1}\right) Z\right] \frac{d x}{x}=i^{n} J_{n}(2 Z) .
$$

Thus

$$
\begin{aligned}
I_{n} & =i^{-2 n} J_{-2 n}(2 Z)-i^{2 n} J_{2 n}(2 Z) \\
& =i^{-2 n}(-1)^{2 n} J_{2 n}(2 Z)-i^{2 n} J_{2 n}(2 Z) \\
& =0 .
\end{aligned}
$$

As a result, $a_{n}(Z)+a_{-n}(Z)=0$.

Given that $i \frac{d a_{0}}{d Z}+a_{1}+a_{-1}=0$, then a direct integration of this relation along with the fact that $a_{1}(Z)+a_{-1}(Z)=0$, leads us to the conclusion $a_{0}(Z)=0$ for every $Z$.
[1] A. L. Jones, J. Opt. Soc. Am. 55, 261 (1965).

[2] D. N. Christodoulides, F. Lederer, and Y. Silberberg, Nature 424, 817 (2003).

[3] N. Stefanou and A. Modinos, Phys. Rev. B 57, 12127 (1998); A. Yariv, Y. Xu, R. K. Lee, and A. Scherer, Opt. Lett. 24, 711 (1999); D. N. Christodoulides and N. K. Efremidis, ibid. 27, 568 (2002).

[4] S. Somekh, E. Garmire, A. Yariv, H. L. Garvin, and R. G. Hunsperger, Appl. Phys. Lett. 22, 46 (1973).

[5] H. S. Eisenberg, Y. Silberberg, R. Morandotti, A. R. Boyd, and J. S. Aitchison, Phys. Rev. Lett. 81, 3383 (1998).

[6] T. Pertsch, U. Peschel, F. Lederer, J. Burghoff, M. Will, S. Nolte, and A. Tünnermann, Opt. Lett. 29, 468 (2004).

[7] J. W. Fleischer, M. Segev, N. K. Efremidis, and D. N. Christodoulides, Nature 422, 147 (2003).

[8] D. N. Christodoulides and R. I. Joseph, Opt. Lett. 13, 794 (1988).

[9] J. Meier, G. I. Stegeman, D. N. Christodoulides, Y. Silberberg, R. Morandotti, H. Yang, G. Salamo, M. Sorel, and J. S. Aitchison, Phys. Rev. Lett. 92, 163902 (2004).

[10] M. A. Ablowitz and Z. H. Musslimani, Physica D 184, 276 (2003).

[11] N. K. Efremidis, S. Sears, D. N. Christodoulides, J. W. Fleischer, and M. Segev, Phys. Rev. E 66, 046602 (2002).

[12] T. Peschel, U. Peschel, and F. Lederer, Phys. Rev. E 57, 1127 (1998).

[13] R. Iwanow, R. Schiek, G. I. Stegeman, T. Pertsch, F. Lederer, Y. Min, and W. Sohler, Phys. Rev. Lett. 93, 113902 (2004).

[14] E. Ultanir, G. I. Stegeman, and D. N. Christodoulides, Opt. Lett. 29, 845 (2004).

[15] A. Fratalocchi, G. Assanto, K. Brzdakiewicz, and M. Karpierz, Opt. Lett. 29, 1530 (2004).

[16] I. Tamm, Phys. Z. Sowjetunion 1, 733 (1932); W. Shockley, Phys. Rev. 56, 317 (1939).

[17] D. Kossel, J. Opt. Soc. Am. 56, 1434 (1966), J. A. Arnaud and A. A. Saleh, Appl. Opt. 13, 2343 (1974).
[18] P. Yeh, A. Yariv, and A. Y. Cho, Appl. Phys. Lett. 32, 104 (1978), W. Ng, P. Yeh, P. C. Chen, and A. Yariv, ibid. 32, 370 (1978); P. Yeh, A. Yariv, and C. S. Hong, J. Opt. Soc. Am. 67, 423 (1977).

[19] M. Miyagi and S. Nishida, Sci. Rep. Res. Inst. Tohoku Univ., 24, 53 (1972).

[20] W. J. Tomlinson, Opt. Lett. 5, 323 (1980).

[21] N. N. Akhmediev, V. I. Korneev, and Y. V. Kuz'menko, Sov. Phys. JETP 61, 62 (1985).

[22] K. G. Makris, S. Suntsov, D. N. Christodoulides, G. I. Stegeman, and A. Hache, Opt. Lett. 30, 2466 (2005).

[23] J. D. Jackson, Classical Electrodynamics, 3rd ed. (Wiley, New York, 1999).

[24] R. Weinstock, Am. J. Phys. 40, 1289 (1970); F. O. Goodman, ibid. 40, 92 (1972); A. H. Nayfeh and M. H. Rice, ibid. 40, 469 (1972).

[25] S. V. Kukhlevsky, G. Nyitray, and V. L. Kantsyrev, Phys. Rev. E 64, 026603 (2001).

[26] M. I. Molina, Phys. Rev. B 71, 035404 (2005).

[27] J. P. Keener, Principles of Applied Mathematics, 3rd ed., (Wiley, New York, 1999).

[28] W. E. Boyce and R. C. DiPrima, Elementary Differential Equations and Boundary Value Problems, 6th ed., (Wiley, New York, 1997).

[29] P. Yeh, Optical Waves in Layered Media (Wiley, New York, 1998).

[30] S. Darmanyan, A. Kobyakov, and F. Lederer, JETP 86, 682 (1998).

[31] E. W. Laedke, O. Kluth, and K. H. Spatschek, Phys. Rev. E 54, 4299 (1996); P. G. Kevrekidis, A. R. Bishop, and K. Ø. Rasmussen, ibid. 63, 036603 (2001).

[32] S. Suntsov, K. G. Makris, D. N. Christodoulides, G. I. Stegeman, A. Hache, R. Morandotti, H. Yang, G. Salamo, and M. Sorel, Phys. Rev. Lett. 96, 063901 (2006).

[33] I. S. Gradshteyn and I. M. Ryzhik, Table of Integrals, Series, and Products, 6th ed. (Academic, New York, 2000). 